\title{
Chinese Patent Medicine As An Adjuvant Treatment for Idiopathic Pulmonary Fibrosis Patients: A Protocol for A Systematic Review and Meta- Analysis
}

\section{Fang Sun}

Beijing University of Chinese Medicine Affiliated Dongzhimen Hospital

\section{Zhaochu Wu}

Beijing University of Chinese Medicine Affiliated Dongzhimen Hospital

\section{Fengli Jiang}

Beijing University of Chinese Medicine Affiliated Dongzhimen Hospital

Mao Huang

Beijing University of Chinese Medicine Affiliated Dongzhimen Hospital Jine Wang

Beijing University of Chinese Medicine Affiliated Dongzhimen Hospital

\section{Wenli Sun}

Beijing University of Chinese Medicine Affiliated Dongzhimen Hospital

\section{Lishan Zhang ( $\square$ Iszhang111@126.com)}

Beijing University of Chinese Medicine Affiliated Dongzhimen Hospital https://orcid.org/0000-00020014-4026

\section{Protocol}

Keywords: Chinese patent medicine, idiopathic pulmonary fibrosis, adjuvant treatment

Posted Date: August 10th, 2021

DOl: https://doi.org/10.21203/rs.3.rs-772721/v1

License: (c) (1) This work is licensed under a Creative Commons Attribution 4.0 International License. Read Full License 


\section{Abstract \\ Background}

Idiopathic pulmonary fibrosis (IPF) is a progressive, chronic, irreversible, and lethal interstitial lung disease (ILD) with unknown etiology occurring mainly in elderly. In recent years, the morbidity and mortality of IPF steady increase. However, there are currently no therapies that reverse fibrosis, the median survival time reported ranging from 2 to 5 years from diagnosis. The purpose of this systematic review and meta-analysis is to evaluate the effectiveness and safety of Chinese patent medicines as an adjuvant treatment in combination with Western medicines in the treatment of patients with IPF.

\section{Methods}

We will search electronic databases including PubMed, EMBASE, Cochrane Central, Web of Science, Chinese Biomedical Database (SinoMed), Chinese National Knowledge Infrastructure (CNKI), Chongqing VIP information (CQVIP), and Wanfang database using keywords related to IPF and Chinese patent medicine. Search was also carried out on ClinicalTrials.gov, International Clinical Trials Registry Platform (ICTRP) and the Chinese Clinical Trials Register (ChiCTR). Reference lists of literature included and relevant systematic reviews will be checked. Two independent reviewers will involved in screening studies, extracting data, and evaluating works. Any disagreements will be resolved by discussion or in consultation with a third reviewer. Data analysis will be conducted using the Review Manager software (version 5.4.1). The risk of bias of each study and the quality of evidence will be respectively assessed using Cochrane Risk of Bias tools and GRADE system.

\section{Discussion}

This review will integrate the current evidence and provide evidence regarding use of Chinese patent medicine as an adjuvant treatment in idiopathic pulmonary fibrosis to provide clarity regarding the potential benefits and best practice.

\section{Trial registration:}

PROSPERO CRD42021252806

\section{Background}

Idiopathic pulmonary fibrosis (IPF) is a progressive, chronic, irreversible, and lethal interstitial lung disease (ILD) with unknown etiology occurring mainly in elderly $(1,2)$. The pathological features of IPF include fibroproliferative foci and excessive deposition of extracellular matrix (ECM). And these histopathological changes are predominantly located in subpleural region(1). Although the precise 
mechanisms of IPF have not been completely understood, it has been revealed that activated fibroblasts and myofibroblasts play a crucial role in regulating tissue repair and ECM secretion during IPF development and are important drivers of structural lung disorders. This ultimately leads to excessive deposition of ECM and irreversible destruction of the lung structure $(3,4)$. Various exposure factors, such as microinhalation, wood dust, viruses and drugs, have been associated with the development of IPF, but the most important risk factor is smoking as many patients with IPF are former or current smokers $(5,6)$.

IPF has a variable and unpredictable clinical course, usually associated with an extremely poor prognosis $(1,7,8)$. Comorbidities of IPF are common in the context of the coexistence of IPF and lung cancer(9). Indeed, the prevalence of lung cancer is much higher in IPF patients. Up to $22 \%$ of patients with IPF will develop lung cancer, with a risk almost five times greater than that of the general population. Moreover, lung cancer significantly worsens the prognosis of $\operatorname{IPF}(10,11)$.In recent years, with a steady increase in the morbidity and mortality of IPF, the prevalence of IPF is estimated to range from 2 to 29 cases per 100,000 population. The median survival time reported ranging from 2 to 5 years from diagnosis $(1,12,13)$.

However, until now for most IPF patients, effective treatments other than lung transplantation remains elusive(1). Non-pharmacological management strategies are applicable to all patients with IPF, regardless of progression or underlying cause. Patients can benefit from smoking cessation, pneumococcal vaccination and annual influenza vaccination, long-term oxygen therapy, and pulmonary rehabilitation $(14,15)$. As for pharmacologic treatment of IPF, the guideline of ATS/ERS/JRS/ALAT only conditionally recommends the use of pirfenidone and nintedanib in patients with IPF(16). The clinical evidence approve that nintedanib and pirfenidone are effective in reducing lung function decline and the risk of exacerbation and death (17-20), but they are not enough to halt Disease progression(21, 22), and accompanied by frequently occurring adverse events(23-25). As there are currently no therapies that reverse fibrosis, the primary goals of intervention are to slow progression, decrease symptoms and improve QOL.

Traditional Chinese medicine(TCM) as a traditional medicine has a history of thousands of years. It treats diseases through therapy with syndrome differentiation. In recent years, a growing body of evidence regarding TCM has demonstrated clinical benefits in IPF patients, proving the improvement effect of TCM on QOL and clinical symptoms with well tolerated and excellent safety profile(26-28). However, TCM compound prescriptions often have a variety and complexity of constituent drugs and dosages. Besides, there are many obstacles to objective evaluation and promotion of their application. Proprietary Chinese medicine refers to ready-to-use medicines made from Chinese herbs and formulated according to certain principles of treatment, such as various kinds of pills, powder, punch, liquor, tincture and ointment. Compared with compounded Chinese medicines, proprietary Chinese medicines have a fixed composition and proportion of drugs and relatively certain dosage, and are therefore easier to use in clinical practice. The purpose of this article is to provide a comprehensive review of the evidence on the efficacy of Chinese patent medicines in combination with western medicines versus western medicine only in the 
treatment of patients with IPF and to explore important directions of future research that should be focused on.

\section{Methods}

This meta-analysis has been prospectively registered in the PROSPERO database (CRD42021252806 Available from: https://www.crd.york.ac.uk/prospero/display_record.php? RecordID=252806). The protocol is prepared based on the the Preferred Reporting Items for Systematic Review and Meta-analysis Protocols(PRISMA-P) statement(29, 30) (Additional file 1). The study started on 27 April 2021 and is scheduled to be completed by 15 September 2022.

\section{Eligibility criteria}

Types of studies. Only randomized controlled trials(RCTs) will be included. Other types of clinical trials and animal trials is not eligible in this study.

Types of participants. The participants were in accordance with the diagnosis of IPF regardless of age, severity, and duration of the diseases, which meet the diagnostic criteria of the consensus of Chinese experts by Chinese Medical Association Respiratory Society or ATS/ERS/JRS/ALAT Clinical Practice Guideline on the diagnosis and treatment of IPF. Patients with other respiratory disease like asthma, bronchiectasia, chronic obstructive pulmonary disease (COPD), and severe liver, kidney, heart disease will be excluded.

Types of interventions. The control group was treated with conventional western medicine treatment modalities, including oxygen therapy, infection control, rehabilitation, and antifibrotic drugs, etc. Patients use acupuncture or other TCM treatment are excluded. Interventions in the experimental group are Chinese patent medicine combine with the same treatment option as the control group. The dosage form and treatment course of Chinese patent medicine are not limited, such as tablet, pill, and injection. Other therapies of TCM like acupuncture, massage, Qigong, are not included in the study.

Types of outcome measures. We will include studies that reported at least one of the following outcomes. IPF is characterized by the deterioration of respiratory functions due to fibrosis of the lung interstitium(1). Progressive deterioration of lung function in patients with IPF is associated with poor prognosis, as lung function declines, QOL follows $(31,32)$. We therefore chose Lung function and life quality as main outcomes, besides, 6-min walking distance (6MWT), blood gas analysis, clinical symptom, adverse events, acute exacerbation (AE), all-cause mortality, and respiratory-specific cause of death or IPF-related mortality as additional outcomes:

\section{Language}

We will include publications and studies reported in the English and Chinese languages. 


\section{Information sources and search methods}

We will perform an all-round search in the following electronic databases from their inception to April 29, 2021: PubMed, EMBASE, Cochrane Central, Web of Science, Chinese Biomedical Database (SinoMed), Chinese National Knowledge Infrastructure (CNKI), Chongqing VIP information (CQVIP), and Wanfang database. Search was also carried out on ClinicalTrials.gov, International Clinical Trials Registry Platform (ICTRP) and the Chinese Clinical Trials Register (ChiCTR) in order to find potential RCTs or data not yet published. If any, references of literature included and previous published systematic reviews and metaanalysis related to TCM and idiopathic pulmonary fibrosis will also be checked. If necessary, We will contact the author to provide additional information. Studies with unavailable data will be excluded. Search is limited to English and Chinese languages. The search will include the following search terms, such as "idiopathic pulmonary fibrosis", "pulmonary fibrosis", "Chinese patent medicine", "Chinese medicine", "traditional medicine". A draft search strategy for PubMed is provided in Additional file 2.

\section{Data management}

All identified records through the performed search will be imported into Endnote X9, and duplicate data will be removed.

\section{Selection of studies}

Two independent reviewers ( $\mathrm{F}$ Sun and $\mathrm{ZC} \mathrm{Wu}$ ) will remove irrelevant articles by reading the title and abstract, and then evaluate the trials to determine if they are appropriate for inclusion by reading the full text. Any disagreement will be resolved by discussion with the third team members (FL Jiang). Full-text articles of eligible studies will be analyzed in detail, and then reviewers will decide which studies to include and explain the reasons for excluding the study. The selection process will be recorded in detail, and the PRISMA flow diagram and tables will be filled in with the characteristics of the included and excluded studies.[17,18]The whole selection process will be presented in a PRISMA flow diagram (33) (Fig. 1).

\section{Data extraction}

Data extraction will undergo a process with independent abstraction by the two reviewers ( $F$ Sun and ZC $\mathrm{Wu}$ ), the final decisions will be made by consensus process. We will check the consistency of information ( $M$ Huang) and disagreements will be resolved by discussion or a third senior reviewer(FL Jiang). Extracted data will include first author, publication year, countries and regions $\llbracket$ participants (age, sex, number of subjects, course of disease $₫$ baseline Information), study design (randomization, allocation concealment, blinding,), trial size, criteria used to screen for IPF diagnosis, interventions and controls (dosage, frequency and duration), outcomes, duration of follow-up, reasons and number of patients who dropped out or lost during follow-up, type and source of financial support and publication status. If required information is incomplete or unclear, we will try to contact the corresponding author of the studies. 


\section{Risk of bias assessment}

We will use the Cochrane collaboration's tool to assess the risk of bias of the included studies, which covers: sequence generation, allocation concealment, blinding, incomplete outcome data (e.g. dropouts and withdrawals) and selective outcome reporting. Two reviewers(JE Wang, WL Sun) will independently assessed risk of bias based on the criteria. Disagreements will be resolved first by discussion and then by consulting a third reviewer(F Sun) for arbitration.

\section{Data synthesis and analysis}

The RevMan (V.5.4.1) software will be used for the meta-analysis. For dichotomous variables, risk ratio (RR) with $95 \%$ confidence interval $(\mathrm{Cl})$ is implemented for statistical analysis. Meanwhile, continuous results will be presented as the weighted mean difference (WMD) or the standardized mean difference (SMD) with $95 \% \mathrm{Cls}$. Under the heterogeneity of the included studies, a random-effects or fixed-effects model will be used. The heterogeneity between the studies will be measured by the $I^{2}$ tests. If the statistical heterogeneity between the results of each study does not exist or is negligible $\left(I^{2}<50 \%, P>0.1\right)$, the fixed-effects model is used for meta-analysis; on the other hand, if there is large statistical heterogeneity detected between the results of the studies $\left(I^{2} \geq 50 \%, P<0.1\right)$, we will try to explain the potential reasons for the differences in heterogeneity by subgroup analysis or sensitivity analysis. After excluding the influence of obvious etiologies of clinical heterogeneity, the random-effects model was used for meta-analysis.

\section{Sensitivity analysis}

Sensitivity analysis will be performed based on risk of bias and analysis method (random-effects model and fixed-effects model) to assess the robustness of our results.

\section{Subgroup analysis}

Subgroup group analysis will be used to explore possible sources of heterogeneity. If necessary, we will try to conduct subgroup analysis based on the different age, severity, course of disease, duration of treatment, drug dose and frequency, dosage form, follow-up period, trial size, risk of bias.

\section{Grading the quality of evidence}

We will use Grading of Recommendations Assessment, Development, and Evaluation (GRADE) system to assess the certainty of evidence for each outcome and entire body of evidence. The quality of evidence will be assessed according to risk of bias, consistency, directness, precision and publication bias. Quality will be adjudicated into four levels: high, moderate, low, or very low. Two reviewers (F Sun, ZC Wu) will independently conduct this assessments. For all phases of the project, reviewers can resolve disagreements by discussion or in consultation with a third reviewer (LS Zhang).

\section{Reporting bias and outcome reporting bias}


Funnel plot or Egger's test to assess the risk of publication bias. Also, we will screen the clinical trial register to evaluate whether selective reporting of outcomes is present.

\section{Ethics and dissemination}

This research is a systematic review and meta-analysis, patients will not be treated directly in the course of the study, and the personal information of patients will not be disclosed. Therefore, informed consent and ethical permission are not required for our research. We intend to update the public registry with this review in all phases of its execution and publish the results in a widely accessible journal after the completion of the research.

\section{Discussion}

IPF is an aggressive form of ILD characterized by chronic progressive fibrosis, with irreversible decline in lung function, progressive respiratory failure and high mortality(34). Evidence suggests that the incidence of IPF is rising(35), however, there are no drugs that can reverse fibrosis yet. Chinese medicine has been used to treat acute and chronic respiratory diseases for more than a thousand years. In recent years, there has been some evidence to support the use of Chinese medicine in the treatment of IPF. The results of several systematic reviews have shown that TCM has a therapeutic effect on IPF(26-28). However, the variable drug composition and dosage of TCM compound formulas make it difficult to replicate and promote clinically. The composition and dosage of proprietary Chinese medicines are relatively fixed, making it easy to promote their application. There have been many clinical studies on the application of proprietary Chinese medicines in the treatment of IPF to assess their effectiveness in the treatment of IPF. However, there are no evidence-based reviews to confirm their efficacy and safety. We will conduct this systematic review and meta-analysis to provide evidence.

We will also analyze the heterogeneity in terms of participant characteristics, dosage form of the drug, treatment regimen, study characteristics and conduct subgroup analyses to identify relevant factors affecting efficacy. A limitation of this review may be that the analysis is limited to studies published in English or Chinese and the quality of the included studies. By integrating and analyzing data from the included studies, we hope to provide high quality evidence on the efficacy and safety of proprietary Chinese medicines for the treatment of IPF, offering a potential treatment option for physicians and patients.

\section{Abbreviations}

AE

acute exacerbation; ChiCTR:Chinese Clinical Trials Register; CNKI:Chinese National Knowledge Infrastructure; COPD:chronic obstructive pulmonary disease; CQVIP:Chongqing VIP information; ECM:extracellular matrix; GRADE:Grading of Recommendations Assessment, Development, and Evaluation; ICTRP:International Clinical Trials Registry Platform; ILD:interstitial lung disease; 
IPF:idiopathic pulmonary fibrosis; TCM:Traditional Chinese medicine; PRISMA:Preferred Reporting Items for Systematic Reviews, Meta-Analyses; PRISMA-P Preferred Reporting Items for Systematic Review and Meta-analysis Protocols; QOL:Quality of life; RCTs:randomized controlled trials; RR:risk ratio; SinoMed:Chinese Biomedical Database; SMD:Standardized mean difference; WMD:weighted mean difference; 6MWD:6-min walking distance; 95\% Cl:95\% confidence interval;

\section{Declarations}

\section{Acknowledgements}

Not applicable.

\section{Authors' contributions}

F Sun and ZC Wu contributed to the design of the study. M Huang, FL Jiang, JE Wang, and WL Sun designed the methods of the systematic review, and LS Zhang will supervise those design and analysis. All authors will be involved in the data extract quality assessment or statistical analysis. Besides, all authors will read and approved the final manuscript.

\section{Funding}

This research received no specific grants from any funding agency in the public, commercial, or not-forprofit sectors.

\section{Availability of data and materials}

The datasets generated during and/or analyzed during the current study are available from the corresponding author on reasonable request.

\section{Ethics approval and consent to participate}

No ethics approval is required because we will use published information.

\section{Consent for publication}

Not applicable.

\section{Competing interests}

The authors declare that they have no competing interests.

\section{Author details}

${ }^{1}$ Dongzhimen Hospital, Beijing University of Chinese Medicine, No.5, Hai Yun Cang, Dongcheng District, Beijing, China 


\section{References}

1. Raghu G, Collard HR, Egan JJ, Martinez FJ, Behr J, Brown KK, et al. An official ATS/ERS/JRS/ALAT statement: idiopathic pulmonary fibrosis: evidence-based guidelines for diagnosis and management. Am J Respir Crit Care Med. [Journal Article; Practice Guideline; Research Support, Non-U.S. Gov't]. 2011 2011-03-15;183(6):788-824.

2. Fanny M, Nascimento M, Baron L, Schricke C, Maillet I, Akbal M, et al. The IL-33 Receptor ST2 Regulates Pulmonary Inflammation and Fibrosis to Bleomycin. FRONT IMMUNOL. [Journal Article]. 2018 2018-01-20;9:1476.

3. Todd NW, Luzina IG, Atamas SP. Molecular and cellular mechanisms of pulmonary fibrosis. Fibrogenesis Tissue Repair. [Journal Article]. 2012 2012-07-23;5(1):11.

4. Selman M, Pardo A. Revealing the pathogenic and aging-related mechanisms of the enigmatic idiopathic pulmonary fibrosis. an integral model. Am J Respir Crit Care Med [Journal Article]. 2014;2014-05-15(10):1161-72. 189(.

5. Wells AU. Managing diagnostic procedures in idiopathic pulmonary fibrosis. Eur Respir Rev. [Journal Article; Review; Systematic Review]. 2013 2013-06-01;22(128):158-62.

6. Wolters PJ, Blackwell TS, Eickelberg O, Loyd JE, Kaminski N, Jenkins G, et al. Time for a change: is idiopathic pulmonary fibrosis still idiopathic and only fibrotic? Lancet Respir Med. [Journal Article; Research Support, N.I.H., Extramural; Research Support, Non-U.S. Gov't; Review]. 2018 2018-0201;6(2):154-60.

7. King TJ, Pardo A, Selman M. Idiopathic pulmonary fibrosis. LANCET. [Journal Article; Review]. 2011 2011-12-03;378(9807):1949-61.

8. Kim HJ, Perlman D, Tomic R. Natural history of idiopathic pulmonary fibrosis. Respir Med. [Journal Article; Research Support, Non-U.S. Gov't; Review]. 2015 2015-06-01;109(6):661-70.

9. Karampitsakos T, Tzilas V, Tringidou R, Steiropoulos P, Aidinis V, Papiris SA, et al. Lung cancer in patients with idiopathic pulmonary fibrosis. PULM PHARMACOL THER. [Journal Article; Review]. 2017 2017-08-01;45:1-10.

10. Tomassetti S, Gurioli C, Ryu JH, Decker PA, Ravaglia C, Tantalocco P, et al. The impact of lung cancer on survival of idiopathic pulmonary fibrosis. CHEST. [Journal Article; Research Support, Non-U.S. Gov't]. 2015 2015-01-01;147(1):157-64.

11. Ozawa Y, Suda T, Naito T, Enomoto N, Hashimoto D, Fujisawa T, et al. Cumulative incidence of and predictive factors for lung cancer in IPF. RESPIROLOGY. [Journal Article]. 2009 2009-0701;14(5):723-8.

12. Wei P, Xie Y, Abel PW, Huang Y, Ma Q, Li L, et al. Transforming growth factor (TGF)-beta1-induced miR-133a inhibits myofibroblast differentiation and pulmonary fibrosis. CELL DEATH DIS. [Journal Article; Research Support, N.I.H. Extramural; Research Support, Non-U.S. Gov't; Research Support, U.S. Gov't, Non-P.H.S.]. 2019 2019-09-11;10(9):670. 
13. Richeldi L, Collard HR, Jones MG. Idiopathic pulmonary fibrosis. LANCET. [Journal Article; Review]. 2017 2017-05-13;389(10082):1941-52.

14. Bell EC, Cox NS, Goh N, Glaspole I, Westall GP, Watson A, et al. Oxygen therapy for interstitial lung disease: a systematic review. Eur Respir Rev. [Journal Article; Meta-Analysis; Review; Systematic Review]. 2017 2017-01-01;26(143).

15. Vainshelboim B, Oliveira J, Yehoshua L, Weiss I, Fox BD, Fruchter O, et al. Exercise training-based pulmonary rehabilitation program is clinically beneficial for idiopathic pulmonary fibrosis. RESPIRATION [Journal Article; Randomized Controlled Trial]. 2014;88(5):378-88. 2014-01-20.

16. Raghu G, Rochwerg B, Zhang Y, Garcia CA, Azuma A, Behr J, et al. An Official ATS/ERS/JRS/ALAT Clinical Practice Guideline: Treatment of Idiopathic Pulmonary Fibrosis. An Update of the 2011 Clinical Practice Guideline. Am J Respir Crit Care Med. [Journal Article; Practice Guideline]. 2015 2015-07-15;192(2):e3-19.

17. Noble PW, Albera C, Bradford WZ, Costabel U, Glassberg MK, Kardatzke D, et al. Pirfenidone in patients with idiopathic pulmonary fibrosis (CAPACITY): two randomised trials. LANCET. [Comparative Study; Journal Article; Multicenter Study; Randomized Controlled Trial; Research Support, Non-U.S. Gov't]. 2011 2011-05-21;377(9779):1760-9.

18. Glaspole I, Bonella F, Bargagli E, Glassberg MK, Caro F, Stansen W, et al. Efficacy and safety of nintedanib in patients with idiopathic pulmonary fibrosis who are elderly or have comorbidities. Respir Res. [Journal Article]. 2021 2021-04-26;22(1):125.

19. Richeldi L, du Bois RM, Raghu G, Azuma A, Brown KK, Costabel U, et al. Efficacy and safety of nintedanib in idiopathic pulmonary fibrosis. N Engl J Med. 2014;370(22):2071-82. [Clinical Trial, Phase III; Journal Article; Multicenter Study; Randomized Controlled Trial; Research Support, Non-U.S. Gov't]... 2014-05-29.

20. King TJ, Bradford WZ, Castro-Bernardini S, Fagan EA, Glaspole I, Glassberg MK, et al. A phase 3 trial of pirfenidone in patients with idiopathic pulmonary fibrosis. N Engl J Med. 2014;370(22):2083-92. [Clinical Trial, Phase III; Journal Article; Multicenter Study; Randomized Controlled Trial; Research Support, Non-U.S. Gov't].. . 2014-05-29.

21. Kolilekas L, Papiris S, Bouros D. Existing and emerging treatments for idiopathic pulmonary fibrosis. Expert Rev Respir Med. [Journal Article; Review]. 2019 2019-03-01;13(3):229-39.

22. Fletcher S, Jones MG, Spinks K, Sgalla G, Marshall BG, Limbrey R, et al. The safety of new drug treatments for idiopathic pulmonary fibrosis. EXPERT OPIN DRUG SAF. [Journal Article; Review]. 2016 2016-11-01;15(11):1483-9.

23. Richeldi L, du Bois RM, Raghu G, Azuma A, Brown KK, Costabel U, et al. Efficacy and safety of nintedanib in idiopathic pulmonary fibrosis. N Engl J Med. 2014;370(22):2071-82. [Clinical Trial, Phase III; Journal Article; Multicenter Study; Randomized Controlled Trial; Research Support, Non-U.S. Gov't].. . 2014-05-29.

24. Costabel U, Albera C, Lancaster LH, Lin CY, Hormel P, Hulter HN, et al. An Open-Label Study of the Long-Term Safety of Pirfenidone in Patients with Idiopathic Pulmonary Fibrosis (RECAP). 
RESPIRATION. [Clinical Trial, Phase III; Journal Article; Multicenter Study]. 2017 2017-0120;94(5):408-15.

25. Cottin V, Koschel D, Gunther A, Albera C, Azuma A, Skold CM, et al. Long-term safety of pirfenidone: results of the prospective, observational PASSPORT study. ERJ Open Res. [Journal Article]. 2018 2018-10-01;4(4).

26. Wu Q, Zhou Y, Feng FC, Zhou XM. Effectiveness and Safety of Chinese Medicine for Idiopathic Pulmonary Fibrosis: A Systematic Review and Meta-Analysis. CHIN J INTEGR MED. [Journal Article; Meta-Analysis; Systematic Review]. 2019 2019-10-01;25(10):778-84.

27. Zhang Y, Gu L, Xia Q, Tian L, Qi J, Cao M. Radix Astragali and Radix Angelicae Sinensis in the Treatment of Idiopathic Pulmonary Fibrosis: A Systematic Review and Meta-analysis. FRONT PHARMACOL. [Systematic Review]. 2020 2020-01-20;11:415.

28. Zhou M, Ye C, Liang Q, Pei Q, Xu F, Wen H. Yiqi Yangyin Huoxue Method in Treating IdiopathicPulmonary Fibrosis: A Systematic Review and Meta-Analysis of Randomized Controlled Trials. Evid Based Complement Alternat Med. [Journal Article; Review]. 2020 2020-0120;2020:8391854.

29. Moher D, Shamseer L, Clarke M, Ghersi D, Liberati A, Petticrew M, et al. Preferred reporting items for systematic review and meta-analysis protocols (PRISMA-P) 2015 statement. Syst Rev. [Journal Article; Research Support, Non-U.S. Gov't; Research Support, U.S. Gov't, P.H.S.]. 2015 2015-01-01;4:1.

30. Shamseer L, Moher D, Clarke M, Ghersi D, Liberati A, Petticrew M, et al. Preferred reporting items for systematic review and meta-analysis protocols (PRISMA-P) 2015: elaboration and explanation. BMJ. [Journal Article; Research Support, Non-U.S. Gov't; Research Support, U.S. Gov't, P.H.S.]. 2015 201501-02;350:g7647.

31. Kistler KD, Nalysnyk L, Rotella P, Esser D. Lung transplantation in idiopathic pulmonary fibrosis: a systematic review of the literature. BMC PULM MED. [Journal Article; Research Support, Non-U.S. Gov't; Review; Systematic Review]. 2014 2014-08-16;14:139.

32. Kreuter M, Wuyts WA, Wijsenbeek M, Bajwah S, Maher TM, Stowasser S, et al. Health-related quality of life and symptoms in patients with IPF treated with nintedanib: analyses of patient-reported outcomes from the INPULSIS(R) trials. Respir Res. [Clinical Trial, Phase III; Journal Article; Randomized Controlled Trial]. 2020 2020-01-30;21(1):36.

33. Page MJ, McKenzie JE, Bossuyt PM, Boutron I, Hoffmann TC, Mulrow CD, et al. The PRISMA 2020 statement: an updated guideline for reporting systematic reviews. BMJ. [Journal Article; Research Support, N.I.H., Extramural; Research Support, Non-U.S. Gov't]. 2021 2021-03-29;372:n71.

34. Barratt SL, Creamer A, Hayton C, Chaudhuri N. Idiopathic Pulmonary Fibrosis (IPF): An Overview. J CLIN MED. [Journal Article; Review]. 2018 2018-08-06;7(8).

35. Hutchinson J, Fogarty A, Hubbard R, McKeever T. Global incidence and mortality of idiopathic pulmonary fibrosis: a systematic review. EUR RESPIR J. [Journal Article; Review; Systematic Review]. 2015 2015-09-01;46(3):795-806. 


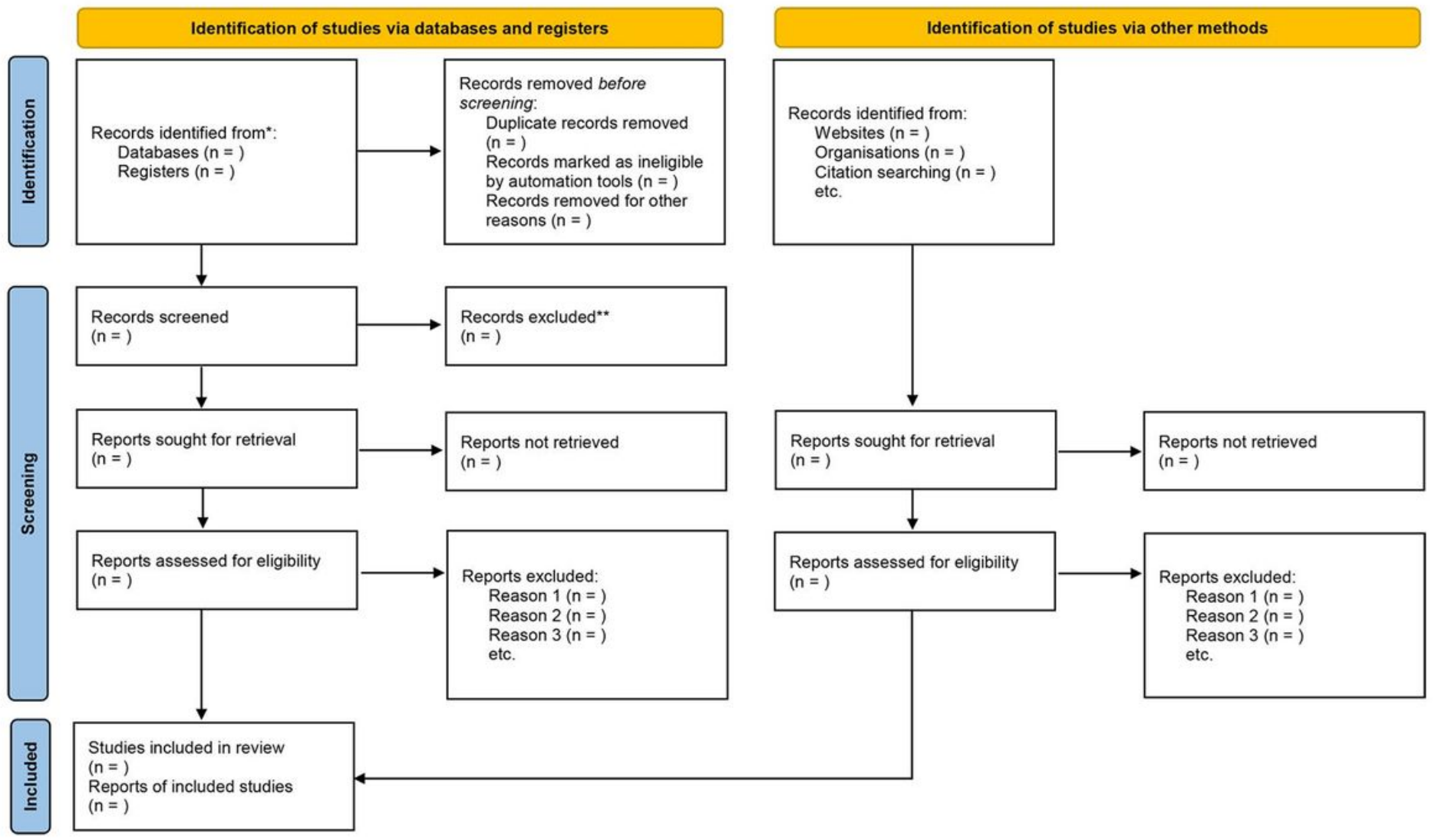

Figure 1

PRISMA 2020 flow diagram for new systematic reviews

\section{Supplementary Files}

This is a list of supplementary files associated with this preprint. Click to download.

- AditionalFile1.docx

- AditionalFile2.docx 